\title{
Multicriteria Decision Making for Evaluation of e-Learning Tools
}

\author{
Eduardo Islas-Pérez, Yasmín Hernández-Pérez, Miguel Pérez-Ramírez, \\ Carlos F. García-Hernández, Benjamín Zayas Pérez \\ Instituto de Investigaciones Eléctricas, Cuernavaca, Morelos 62490, \\ Mexico \\ \{eislas, myhp, mperez, cfgarcia, zayas\}@iie.org.mx
}

\begin{abstract}
This document is in the required format. This work shows a benchmark of e-Learning tools including an approach for comparing them based on histogram specification concepts. The analysis is based on the definition of a set of criteria which are useful and desirable characteristics of learning management systems. The final results show the evaluation from different views including the approach based on their histograms. The evaluation of each e-Learning tool is based on the use of a three-dimensional model which organizes the criteria in three different axes according to their functionality inside the model, namely: Management, Technological and Instructional. With the application of the evaluation methodology we can assess the tools from different points of view. One of the main objectives of this work is to help users and developers of eLearning tools to make good decisions about which tool have the best features for developing training and learning systems and for development and management of resources, courses and learning objects.
\end{abstract}

Keywords: e-Learning, evaluation methodology, learning management systems, multi-criteria decision making.

\section{Introduction}

The aim of this work is to present updated outcomes of a benchmarking of e-Learning technologies which is based on a proposed evaluation methodology within a threedimensional model of criteria (3D model). The information, the evaluation methodology and the 3D model of criteria might provide useful information to eLearning users and developers to make good decisions about which tool has or should have the best features for choosing or developing a management system of instructional resources such as courses and learning objects.

The proposed methodology in this work is very useful to evaluate the applicability of each learning tool from a global point of view as well as to establish the ranking of each learning tool in different standpoints.

Commercial and free LMSs, integrate different modules providing a complete learning tool. However, this integration increases the cost and complexity of each tool. 
In this accomplishment we present evaluation results for eight Learning Management Systems.

Although the extant literature has many articles, books, internet services, and guides to evaluate LMS packages $[1,2]$; they do not use the approach presented in this work, and where there is some similarity, the method is not described in detail as it is covered here. The evaluation methodology described can be used to evaluate other kind of items, using office tools and it can be adapted to evaluate other software products as Database Management Systems [3] or others. We have also applied the methodology to evaluate Virtual Reality development tools and even Virtual Reality equipment [4].

Nowadays an increasingly huge number of LMS packages are available; more than 165 are mentioned in [5] where it is also shown an evolution of several tools from open source platforms to commercial platforms.

The proposed methodology was used to update the evaluation of only five commercial platforms (Docebo, Joomla, Blackboard, IBM Social Learning and PeopleSoft) and three open source tools (Dokeos, Moodle and Sakai) since these LMS are still extensively used since our last evaluation described in [6]. Some of these tools (IBM Social Learning and PeopleSoft) are not LMS strictly but have some functionality related with e-Learning and e-Training, for instance they allow to load and publish instructional content, also allow asynchronous communication, etc. We believe that this evaluation although uneven for these tools might be useful for companies to make decisions about which tool fulfill their requirements to use in their e-Learning and eTraining activities [7].

The rest of this work is organized as follows: some related work is presented in section 2; section 3 describes the evaluation methodology; section 4 illustrates how the methodology was applied to evaluate different e-Learning tools from different perspectives including an approach for equating each tool based on histogram specification with respect to a sound e-Learning tool; finally section 5 provides some conclusions.

\section{Related Work}

The set of criteria in the 3D-model is based on [8], the main differences are how we apply them in evaluation: we assign several characteristics to each criterion and give different weights to each of them based on their relevance and we also group them in the 3-dimensional model in order to evaluate the tools from different perspectives such as instructional, management and technological.

Regarding evaluation methods, there are different approaches [9]; one of them distinguishes quantitative and qualitative methods. The former gives numerical results and the later use narrative or descriptive data rather than numbers [10].

Within the quantitative methods, one of the most used is the MultiCriteria Decision Making (MCDM) [11], [12]. At the core of the MCDM, a list of criteria must be defined; each criterion specifies a parameter to be evaluated, since they personalize a specific feature of the item under evaluation.

The MCDM are general purpose methods, in the sense that depending on the kind of items to be evaluated, they demand the definition of a specific set of criteria (or 
parameters) that an item in turn must accomplish with. The set of criteria in turn personalizes the methodology and at the same time makes it flexible enough to be applied in the evaluation of different kinds of items. However the sets of steps involved in a methodology might remain unaltered. That is, the criteria are different but the methodology is the same. This flexibility makes MCDM a powerful methodology with a large range of application. There is even a conference only in this topic and with this name MCDM [13]. This methodology has applications in Constructive Preference Learning in MCDA (Multiple Criteria Decision Aiding), Infrastructure Planning and Environmental Management, MCDA Models in Risk, Reliability and Maintenance Contexts, MCDM for smart and sustainable communities, among others [13].

We do have already used MCDM methodology to evaluated different kinds of items such as LMSs, Virtual Reality development tools, different types of hardware, etc., as long as we establish an appropriate set of criteria in each case.

\section{Evaluation Methodology}

In the next four subsections the complete methodology is described in several stages from criteria selection (i), until deployment of results and conclusions (vii)

\subsection{Three-Dimensional Model Figures}

The model relates the three most important aspects involved in personnel training and that constitutes the three axes of the 3D model, namely: Management $(\mathrm{M})$, Technological (T) and Instructional (I) axes; these aspects allow three combinations between two axis (MT, MI, TI); and the combination of all of them (MTI). Accordingly this provides different viewpoints which allow evaluating each tool from seven different perspectives; these perspectives help to determine whether or not a tool fulfills the requirements from a Management, Technological or Instructional point of view.

The management dimension is related with administrative features of the tool and we can evaluate aspects such as: student tracking, curriculum management, statistics, etc. The instructional dimension deals with features related with instructional design, didactic planning, content production, instructor manual, student manual, and so on. Finally, the technological dimension involves attributes related to software and hardware tools used in the learning processes.

These three conceptual dimensions (axes) outline a 3D space and three planes, see Figure 1.

Each axis represents a set of attributes, so that the planes and the space represent different combinations of attributes of the axes involved. The attributes and combinations of attributes are shared by each criterion grouped in these planes, axes and the space. For instance, the criteria in the management-technological plane are helpful to determinate different management aspects made possible by technology. Thus, for example, instructors might be able to record delivery and review assignments of students online. This is possible because Learning Management Systems (LMS) are network platforms. Finally, the management-technological-instructional space indicates how the technology is being used to manage the learning process. 


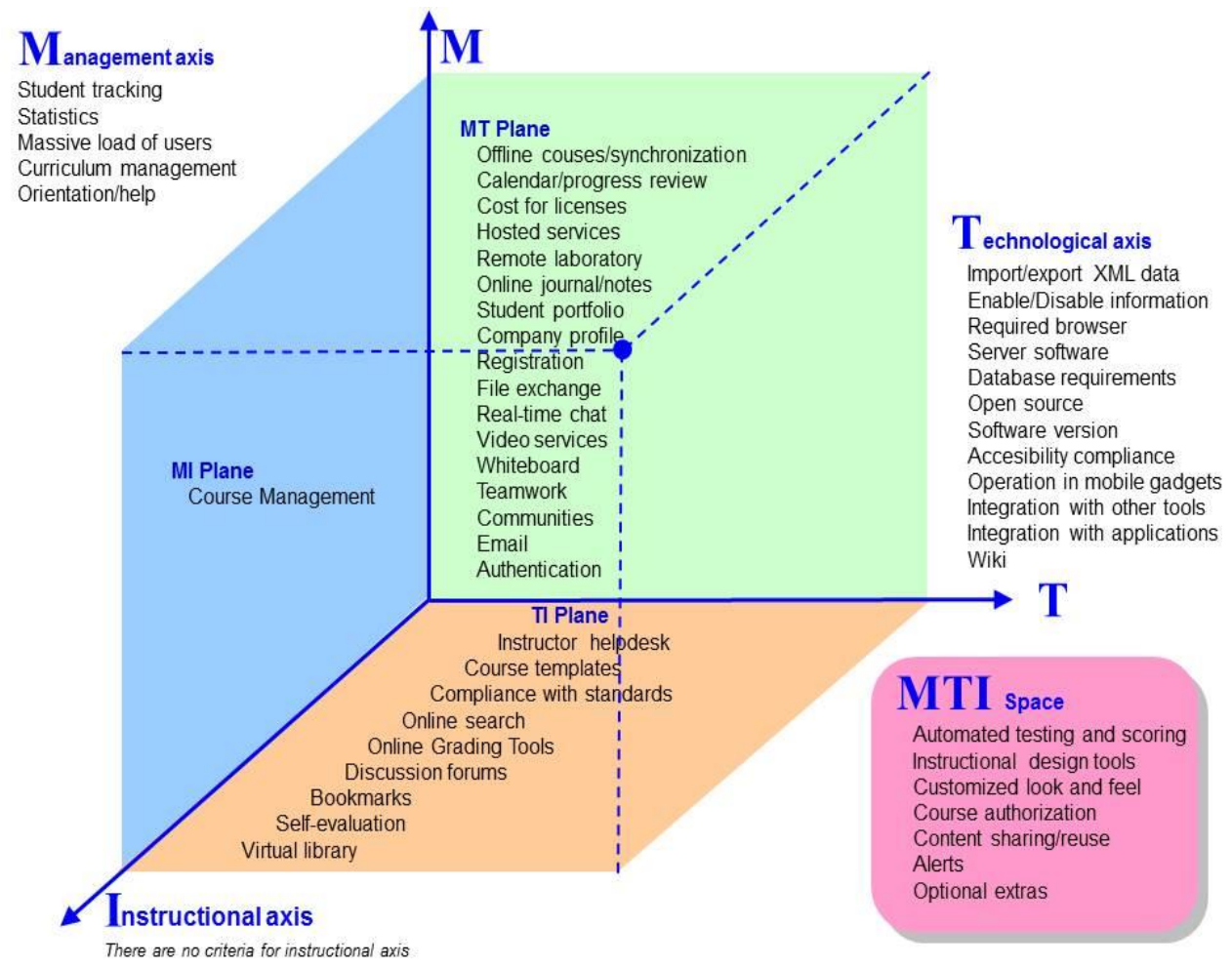

Fig. 1. Three-dimensional model (3D model) to evaluate modern learning and training systems.

\subsection{Criteria and Weight Definition}

The methodology is based on 51 criteria used to evaluate different technologies applied in modern training and learning systems. The same methodology was applied in our previous evaluation of e-Learning tools made in 2007 [6]. The criteria for e-Learning tools are grouped in the 3D model described above in accordance with their use and application in training and learning processes.

The methodology includes seven steps. This approach has been already applied successfully in $[3,6]$ :

i. Criteria selection. Once we know the kind of item to evaluate (LMSs in this case), in this step a group of criteria is defined. Each criterion will evaluate the degree of the item accomplishment of some requirement imposed by the user.

ii. Scales definition. A scale from 0 to 5 is assigned to every criterion; the scale will be useful to locate the degree of accomplishment of the criterion in turn by the tool in evaluation.

iii. Weight assignment. The importance of every criterion by assigning weights which range between 1 and 2 is defined. Thus, the number 2 is assigned to 
those criteria which according to evaluator are more important for the company. On the other hand 1 is assigned, for instance, to needed criteria but being less important for the company. The purpose of the hierarchy is to make weighting easier; in theory, how it is structured should not affect the final weight assigned for each criterion [14, 15]. In experiments carried out in [14] non-hierarchical weights tend to be "flatter" (more equal), while hierarchical weights are "steeper" (have a greater variance).

iv. Selection of the items to evaluate. A set of specific items to be evaluated should be selected. This selection depends on the purpose of the items and on the evaluation itself. The criteria in turn represent the end users requirements.

\subsection{Definition of Evaluation Methods}

The evaluation study reported in [6], shows that three different [7, 14-17] Multi-Criteria Decision Making (MCDM) applied to the evaluation of LMSs was consistent, here is shown the additive value function and non-hierarchical weight assessment method (NWAM).

v. Analysis and evaluation of each LMS. Based on the criteria and the weight assignment, the tools were reviewed, analyzed and evaluated, grading them in accordance with the method NWAM:

MCDM: Additive value function and non-hierarchical weight assessment.

where:

$$
\operatorname{MAXV}\left(A_{i j}\right)=\sum_{i=1}^{n} w_{i} v_{i}\left(x_{i j}\right)
$$

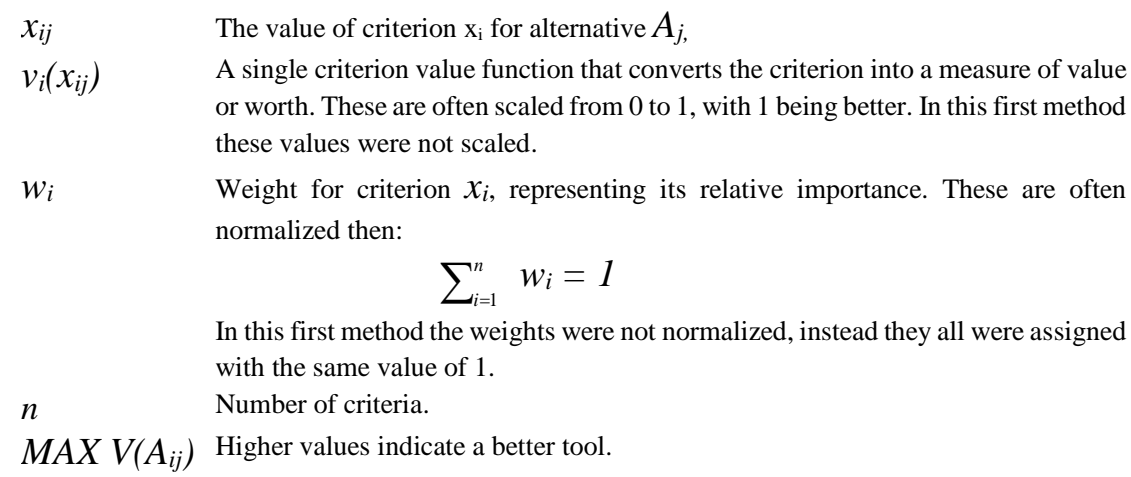

\subsection{Results}

vi. Comparison of LMSs. In this step the results obtained are represented in different charts, considering each axis, plane, space, their histograms and the whole evaluation. These charts provide the means to compare them so that we 
can establish graphically strengths and weaknesses of the different tools under evaluation.

vii. Results obtained and conclusions. Based on all previous outcomes, finally we can draw some conclusions and the numerical outcomes will provide support to the decision making. Program listings or program commands in the text are normally set in typewriter font, e.g., CMTT10 or Courier.

\section{Application of the Evaluation Methodology}

Here is shown how the methodology is used to evaluate different LMSs.

\subsection{Criteria Definition and Value Assignment}

In Figure 1 the whole set of criteria are shown. Some criteria were taken from [8] and were grouped for each dimension (the criteria that involve only one dimension), in each plane (two dimensions) or in space (three dimensions).

As we stated in [6] "Although subjective, it is worth clarifying that this grouping is based on our experience and the criteria could be grouped in a completely different way, for instance some criteria can be included in a plane or in the space. For instance student tracking was classified as M (because student tracking usually is a Management activity) but it could have been classified in the MT plane (because this kind of management is achieved using LMS technology). It depends on the significance for users and developers on different aspects that a criterion might involve". Nevertheless the overall score of the evaluation of an LMS remains unchanged, no matter where the criteria are located, in an axe, in a plane or in the space.

That is to say, with the obtained results in this and our previous work [6] and since each tool is assigned the total sum of values of all criteria we can establish that axes, planes and space are going to provide us with detailed different approaches or views of the evaluations but the overall evaluation is constant.

In this work, only one criterion is described in detail, as well as its value assignment (Table 1). The rest of the criteria were analyzed in the same way and are described in detail in [6].

Likewise in [8] additionally to the evaluation, other activities involved in the analysis of modern training systems can be integrated to the 3D model or complementing it, for example:

i. A cost - benefit analysis might be needed so that a company would be able to make decision on purchasing, development or using an e-Learning tool. These in turns might involve time and technical support considerations.

ii. Correlate the company competences and the solution of specific problems that the enterprise faces.

iii. Take into account practical guidelines to optimize the use of technologies for instructional purposes.

iv. Etc. 
Table 1. Student tracking criterion: Value assignment.

\begin{tabular}{ll}
\hline Features \\
\hline 1. & Only the tracking of exams can be carried out. \\
2. & Every element can be selected to carry out the student's tracking (homework, tests, \\
essays, final exam, projects, etc.) \\
3. & Emissions of reports of every element in the course. \\
4. & Different reports can be selected. \\
5. & The reports can be configured to present one or several elements at the same time. \\
\hline Scale & Description \\
\hline $\mathbf{0}$ & Student tracking is not supported. \\
\hline $\mathbf{1}$ & The tool has one of the features above \\
\hline $\mathbf{2}$ & The tool has two of the features above \\
\hline $\mathbf{3}$ & The tool has three of the features above \\
\hline $\mathbf{5}$ & The tool has four of the features above \\
\hline
\end{tabular}

\subsection{LMSs Evaluation Results}

The following subsections show an update of the results published previously in [6]. In this actualization are depicted the evaluations of the systems: Blackboard, Docebo, Dokeos, IBM Social Learning, Joomla, Moodle, PeopleSoft and Sakai. We chose these tools trying to include the most popular LMSs and learning tools. However they will be helpful to illustrate the use of the 3D model as an evaluation methodology.

\subsubsection{Results Obtained for e-Learning Systems from Different Perspectives}

The evaluation was carried out by assessing the degree of fulfilment of features of each criterion. Some e-Learning tool was tested and evaluated in collaboration with software providers where each feature of each criterion was reviewed. The results for all perspectives except for the Instructional axis (because it does not have any criteria associated in our approach) are presented in a graphical way in Figures 2, 3, 4, 5, 6 and 7.

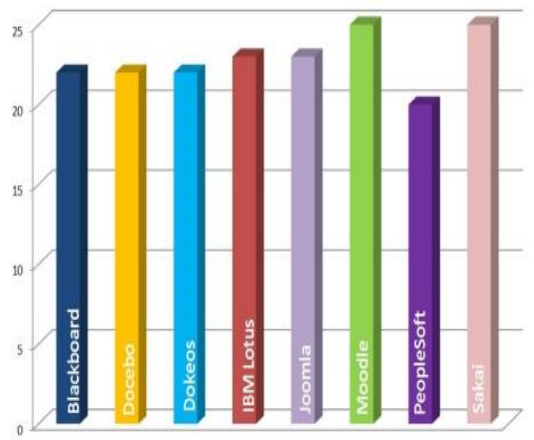

Fig. 2. Management axis.

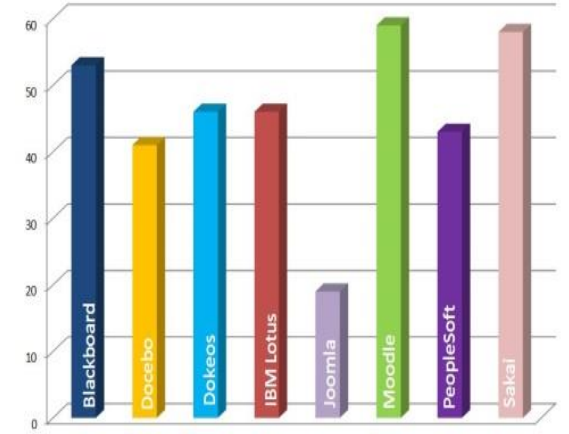

Fig. 3. Technological axis. 


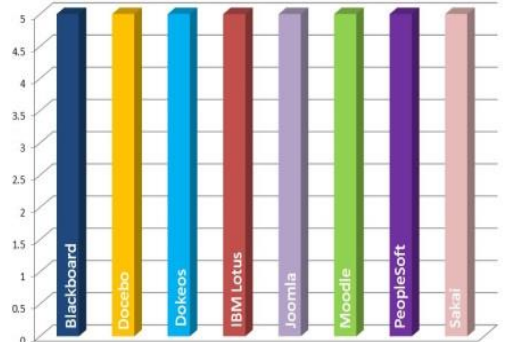

Fig. 4. Management-Instructional plane.

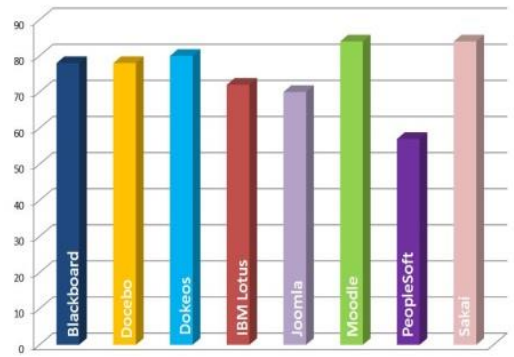

Fig. 6. Management-Technological plane.

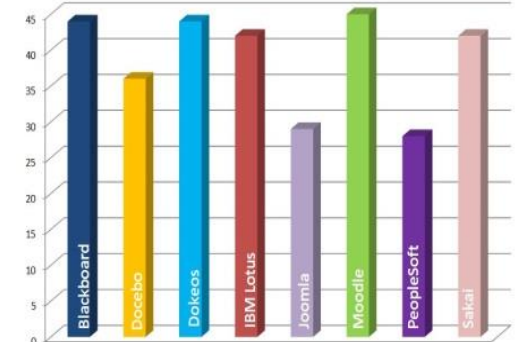

Fig. 5. Technological-Instructional plane.

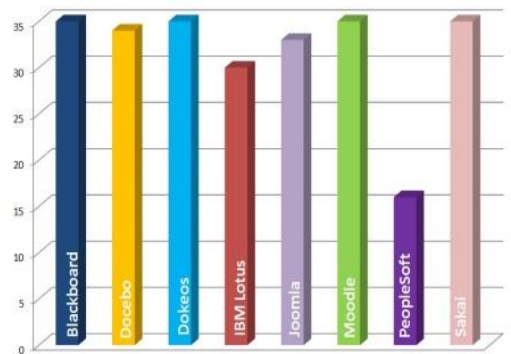

Fig. 7. Three-dimensional space (MTI).

\subsubsection{Outcomes from Applying the MCDM}

The results for the first MCDM method are depicted in Figure 8, which shows the ranking and global results for each software tool. These global results include all the criteria considered applying the additive value function without scaling the value function $V_{i}\left(\mathrm{X}_{i j}\right)$ and using non-hierarchical weight assessment. In this method, the best evaluated tool was Moodle followed by Sakai.

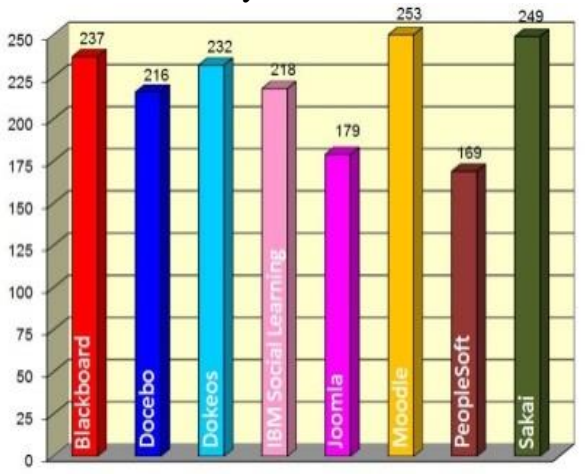

Fig. 8. Total sum of values using the MCDM.

Considering the results obtained in our previous work [6] and corroborating the grades of this actualization we can compare the methods and rules in terms of their ease of use, appropriateness and validity as it was stated in $[6,11,12]$. 
Ease of Use and Appropriateness.

Once the set of criteria and the weights for each criterion have been defined, to follow the MCDM method described above does not represent a problem. At most it will demand some time depending on the number of criteria. In [6] was shown that no matter what MDCM is used to evaluate, the outcomes are consistent.

\subsubsection{Comparison of Results Using Histogram Matching and Histogram Specification}

In the digital image processing area there is the concept of histogram equalization to produce an output image that has a uniform histogram. This output image features a good quality and worthy contrast. Also in this area the concept of histogram specification is useful to be able to specify the shape of the image histogram that we wish to accomplish. The method used to generate a desired output that has a specified histogram is called histogram matching or histogram specification [18].

In this section we use these concepts and present the obtained histogram for each eLearning tool taking into account all the criteria (see Fig. 1). See the assessments shown in Figure 9.

The histogram allows having a general view about the degree of accomplishment of each criterion by the set of LMSs evaluated. Thus, we can see that Remote laboratory is the lowest accomplishment criteria by all the LMS evaluated. On the other hand we can observe that criteria such as Required browser, Discussion forums, Authentication, etc. are well accomplished by all LMSs evaluated.

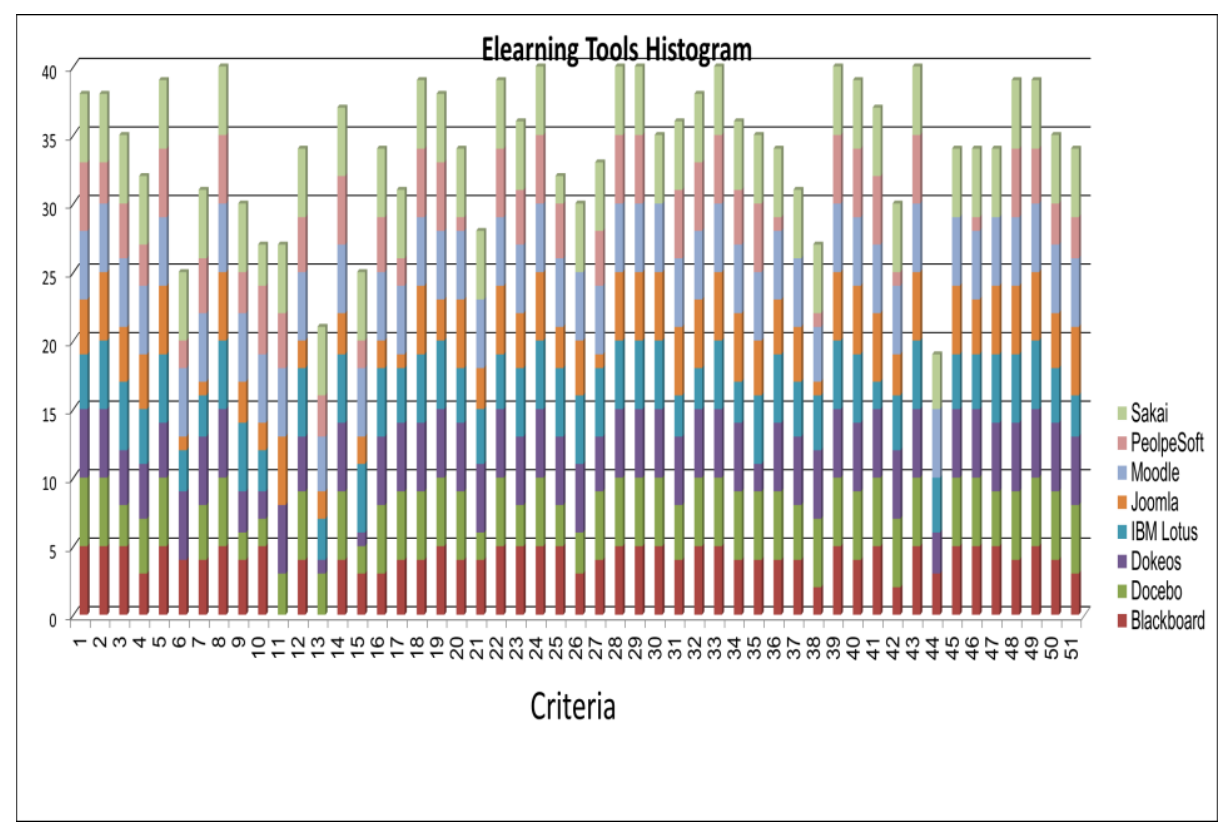

Fig. 9. E-Learning tools histogram, the numbered criteria are shown in Table 2. 
Table 2. Set of criteria (based on [8])

\begin{tabular}{|c|c|c|c|c|c|}
\hline No & Criterion & No & Criterion & No & Criterion \\
\hline 1 & Student Tracking & 18 & Course Management & 35 & Real-Time Chat \\
\hline 2 & Statistics & 19 & Instructor Helpdesk & 36 & Video Services \\
\hline 3 & Massive load of users & 20 & Course Templates & 37 & Whiteboard \\
\hline 4 & Curriculum Management & 21 & $\begin{array}{l}\text { Compliance with } \\
\text { Standards }\end{array}$ & 38 & $\begin{array}{l}\text { Offline } \\
\text { Courses/Synchronization }\end{array}$ \\
\hline 5 & Orientation/Help & 22 & Online Search & 39 & Teamwork \\
\hline 6 & Import/Export XML data & 23 & Online Grading Tools & 40 & Communities \\
\hline 7 & Enable/Disable information & 24 & Discussion Forums & 41 & Student Portfolio \\
\hline 8 & Required Browser & 25 & Bookmarks & 42 & Cost of licenses \\
\hline 9 & Server Software & 26 & Self-evaluation & 43 & Company Profile \\
\hline 10 & Database Requirements & 27 & Virtual Library & 44 & Remote Laboratory \\
\hline 11 & Open Source & 28 & $\begin{array}{l}\text { Calendar/Progress } \\
\text { Review }\end{array}$ & 45 & $\begin{array}{l}\text { Automated Testing and } \\
\text { Scoring }\end{array}$ \\
\hline 12 & Software Version & 29 & Authentication & 46 & Instructional Design Tools \\
\hline 13 & Accessibility Compliance & 30 & Hosted Services & 47 & Customized Look and Feel \\
\hline 14 & Operation in Mobile Gadgets & & Registration & 48 & Course Authorization \\
\hline 15 & Integration with other Tools & 32 & File Exchange & 49 & Content Sharing/Reuse \\
\hline 16 & Integration with applications & 33 & Email & 50 & Alerts \\
\hline 17 & Wiki & 34 & On-line Journal/Notes & & Optional Extras \\
\hline
\end{tabular}

\section{Conclusions}

In the application of MCDM methods to make a decision based on the results, Hobbs and Meier [9] recommend to apply more than one approach because different methods offer different results to compare. In evaluating the results of different methods, the potential for biases should be kept in mind. The extra effort is not large and the potential benefits, in terms of enhanced confidence and a more reliable evaluation process, are worth. However the results shown in our previous work [6] and in this accomplishment deploy the same ranking of choices it does not matter the method used as opposed in [13]. The model can be used to analyze a broad variety of different e-Learning technologies.

The main benefits obtained with the evaluation of several e-Learning tools from a general perspective and from different points of view including the approach for equating tools with respect to a sound software tool are: (1) personnel related in evaluating and selecting an appropriate tool is now informed about the differences and accomplishment of each tool and (2) e-Learning firms can identify the opportunity areas and features where they can improve their tools. The decision for choosing an eLearning tool can be made taking into account: management, technological and instructional characteristics. Furthermore, decision makers can make up an action plan and choose the best path to follow in order to integrate this technology into their learning and training processes. 


\section{References}

1. Bill Brandon: 311 Tips for the Successful Management of an LMS or LCMS. 1st ed. USA: The e-Learning Guild, 42 p. (2006)

2. WCET Learn. A Guide for Planning Communications during LMS Selection, Implementation and Beyond. [Online] (2010)

3. Eduardo Islas, Eric Zabre, Miguel Pérez: Evaluación de herramientas de hardware y software para el desarrollo de aplicaciones de realidad virtual. Bulletin Electrical Research Institute, 28:61-67 (2004)

4. Miguel Pérez, Eric Zabre and Eduardo Islas: Prospectiva y ruta tecnológica para el uso de la tecnología de realidad virtual en los procesos de la CFE. Instituto de Investigaciones Eléctricas, Cuernavaca, México (2004)

5. e-Learning Industry. Learning Management Systems Comparison Checklist of Features. [Online] Available from: http://e-Learningindustry.com/learning-management-systemscomparison-checklist-of-features [Accessed: 02/09/2015] (2013)

6. Eduardo Islas, Miguel Pérez, Guillermo Rodríguez, Israel Paredes, Ivonne Ávila, Miguel Mendoza: E-Learning Tools Evaluation and Roadmap Development for an Electrical Utility. Journal of Theoretical and Applied Electronic Commerce Research, 2(1):63-75 (2007)

7. William Horton, Katherine Horton: E-Learning Tools and Technologies: A consumer's guide for trainers, teachers, educators, and instructional designers. 1st ed., Indianapolis, USA, Wiley Publishing, 592 p. (2003)

8. Edutools: CMS Home, Edutools. [Online], Available: http://www.edutools.info/course/ (2013)

9. Aravossis Konstantinos, Koutsiana Efrosini: Program Evaluation Methodologies. A comparative Assestment. Discussion paper series, 9(17): 387-404 (2003)

10. APCRC: Evaluation methodology. APCRC, Bristol (2015)

11. B. F. Hobbs, P. M. Meier: Multicriteria Methods for Resource Planning: An experimental comparison. IEEE Transactions on Power Systems, 9(4): 1811-1817 (November 1994)

12. Benjamin F. Hobbs, Vira Chankong, Wael Hamadeh, Eugene Z. Stakhiv: Does Choice of Multicriteria Method Matter? An experiment in Water Resources Planning. Water Resources Research, 28(7):1767-1779, DOI: 10.1029/92WR00712 (1992)

13. MCDM: 23rd International Conference on Multiple Criteria Decision Making MCDM 2015 - Bridging Disciplines, http://www2.hsu-hh.de/logistik/MCDM-2015/invitedsessions.html

14. William G. Stillwell, Detlof von Winterfeldt, Richard S. John: Comparing Hierarchical and Nonhierarchical Weighting Methods for Eliciting Multiattribute Value Models. Management Science, 33(4):442-450 (1987)

15. Michelle L. Bell, Benjamin F. Hobbs, Emily M. Elliott, Hugh Ellis, Zachary Robinson: An evaluation of multi-criteria methods in integrated assessment of climate policy. Journal of Multi-Criteria Decision Analysis, 10(5):229-256, DOI: 10.1002/mcda.305 (2002)

16. Vira Chankong, Yacov Y Haimes: Multiobjective Decisión Making: Theory and Methods. Amsterdam, North-Holland, 432 p. (1983)

17. Theodor Stewart: A Critical Survey on the Status of Multiple Criteria Decision Making Theory and Practice. The International Journal of Management Science, 20(5-6):569-586. DOI: 10.1016/0305-0483(92)90003-P (1992)

18. Rafael C. Gonzalez, Richard E. Woods: Digital Image Processing. 3rd ed., New Jersey, USA, Pearson, Prentice Hall, 954 p. (2008)

19. M. Baldonado, C.-C.K. Chang, L. Gravano, A. Paepcke: The Stanford Digital Library Metadata Architecture. Int. J. Digit. Libr., 1:108-121 (1997) 